\title{
Attentional bias affects change detection
}

\author{
RICHARD H. YAXLEY and ROLF A. ZWAAN \\ Florida State University, Tallahassee, Florida
}

\begin{abstract}
Thirty smokers and 30 nonsmokers participated in a flicker study in which the role of attentional bias in change detection was examined. The participants observed picture pairs of everyday objects flicker on a computer screen until they detected the one object that had changed. In half of the pictures, a smoking-related object (e.g., a lighter) was included among smoking-unrelated objects (e.g., a spoon). Half of the smokers and half of the nonsmokers were aware of the experiment's focus, and the other half were not. The smokers exhibited shorter detection latencies than did the nonsmokers when a smoking object changed and longer detection latencies when a nonsmoking object changed while a smoking object was present, and they exhibited detection latencies similar to those of the nonsmokers when smoking objects were not present. Interestingly, the nonsmokers displayed the same attentional bias as the smokers when they were aware of the experiment's smoking focus, but they did not display any attentional bias when they were unaware. Thus, these findings provide evidence for long-term context-independent, as well as for short-term context-dependent, attentional bias.
\end{abstract}

Operating effectively within one's environment requires continual monitoring of the presence, location, and identity of objects. Attention plays a critical two-sided role in such monitoring; it can be attracted to the presence of extrinsic objects or features (e.g., motion or color) or directed by intrinsic biases to particular types of objects or features. Studies on addiction suggest that drug users acquire attentional biases for drug-related objects that lead to highly individualized appraisals of subjectively relevant stimuli (Ryan, 2002). Such biases influence the degree to which an object is evaluated, given one's individualized goals. For example, Tiffany (1990) proposed that a drug user's goals are appointed from repeated interactions with drug-related objects. Over time, these routine interactions lead to the formation of automatized action plans. These action plans are enacted with exposure to drug-related objects, and any controlled attempt to impede such action plans consumes attentional resources that would otherwise be available for other types of processing. Thus, one's goals and level of experience influence how one evaluates the environment.

For example, when tobacco smokers are exposed to smoking-related objects and are unable to perform their smoking ritual, they exhibit an attentional bias that reduces those attentional resources that would otherwise be available for other tasks, such as simple tone reaction (Sayette \& Hufford, 1994), language processing (Zwaan \& Truitt,

This research was supported by Grant DA 11678 from the National Institute on Drug Abuse to R.A.Z. We thank Mark Aveyard and Carol Madden, as well as Anne Hillstrom, Andrew Hollingworth, Barry Jones, and an anonymous reviewer, for helpful comments on previous versions of the manuscript. Correspondence regarding this manuscript should be addressed to R. A. Zwaan, Department of Psychology, Florida State University, Tallahassee, FL 32306-1270 (e-mail: zwaan@psy.fsu.edu).
1998, 2000), and mathematical problem solving (Madden \& Zwaan, 2001). Similarly, studies in which the visual probe paradigm has been used have demonstrated that smokers exhibit an attentional bias by directing their attention to depictions that include a smoking-related object (Ehrman et al., 2002; Mogg, Bradley, Field, \& Houwer, 2003). Alcohol and cannabis users have also exhibited attentional biases to alcohol- and cannabis-related objects, so that these types of objects influence their ability to detect visual change (B. C. Jones, B. T. Jones, Blundell, \& Bruce, 2002; B. T. Jones, B. C. Jones, Smith, \& Copley, 2003).

Detecting visual change in one's environment is a task engaged in routinely. And given the richness of visual perception and the general success of interacting with objects in a dynamic environment, observers may often have an inflated sense of how much visual information they are monitoring at any given point. An increasing amount of evidence suggests that observers may be monitoring far less information than is commonly believed (O'Regan, 1992; O'Regan \& Noë, 2001). For instance, a number of empirical studies have demonstrated that people are often "blind" to changing elements in scenes (for reviews, see Rensink, 2000, 2002; Simons \& Levin, 1997). One such phenomenon, termed change blindness (CB), occurs when large-scale scene changes go unnoticed when they are synchronized with global visual disruptions, such as eye movements (McConkie \& Currie, 1996) or film cuts (Levin \& Simons, 1997) or when two pictures of a changing scene are flickered (Rensink, O'Regan, \& Clark, 1997).

Evidence suggests that the ability to detect change is influenced by how attention is directed, both when attention is attracted to an object because it has certain features and when the direction of attention is driven by contextual knowledge. As evidence that stimulus characteristics can 
attract attention to changes, Scholl (2000) demonstrated that when the change to be detected was an abrupt, late onset of an object, the salience of that onset overrode any conceptual influences on how attention would normally have been directed in the scene. However, Rensink et al. (1997) clearly demonstrated that observers' background knowledge of depicted events directed attention to those objects in the scene that were most relevant.

Using the flicker paradigm, Rensink et al. (1997) presented participants with a flickering of two pictures of a scene that included a change with respect to an object's presence, location, or color. Typically, such rapid visual changes create a motion signal, which attracts attention. The presentation of a visual mask was interleaved between the flickering pictures to eliminate the motion signal. When scene changes were presented in this manner, observers found the changes difficult, if not impossible, to detect. Of relevance to the present article, the ability to detect changes was found to be modulated by background knowledge. After having an initial group of observers describe the events depicted in the scenes, Rensink et al. identified areas of central interest as those areas that were mentioned by a majority of the participants, and those areas that were not mentioned by the observers were identified as areas of marginal interest. Rensink et al. discovered that the centrality of the change to the depicted event affected observers' detection of change, so that changes in central areas were more readily noticed than were changes in marginal areas of interest.

B. T. Jones et al. (2003) employed the flicker paradigm to investigate the influence of alcohol- and cannabisrelated objects on change detection. B. T. Jones et al. demonstrated that drug users exhibited an attentional bias for drug-related objects, so that heavier users detected changes in drug-related objects more quickly and in neutral objects more slowly than did lighter users and nonusers. B. C. Jones et al. (2002) provided converging evidence that attentional biases influence change detection when multiple objects (drug related vs. neutral) undergo simultaneous change. These studies proposed that the biases in heavier alcohol and cannabis users augment the detection of changing alcohol- and cannabis-related objects by directing attention to those objects when they change. However, the same biases attenuate the detection of changing neutral objects by continuing to direct attention to the alcohol- and cannabis-related objects.

As the above studies suggest, knowledge and biases play an important role in directing our attention toward or away from changing objects in scenes. However, several questions remain unresolved. First, it is important to distinguish between attentional biases that are enduring and task independent and attentional biases that are short term and task dependent. The first bias presumably arises as a result of experience (e.g., with the use of a certain drug), whereas the second is presumably prompted by the participant's understanding of the task. First, it needs to be determined whether and to what extent each of these biases affects change detection. Second, it needs to be established that the attentional bias effect on change detection generalizes to other drugs. Third, it needs to be determined to what extent the flicker paradigm can be modified (e.g., number of trials, spatial distribution of objects) and remain sensitive to attentional biases. In the studies by B. C. Jones et al. (2002), and B. T. Jones et al. (2003), attentional bias was demonstrated to influence performance across a single trial in which the groupings of drug-related and neutral objects were segregated to the opposite sides of the scene. Thus, it remains to be determined whether attentional biases persist across multiple trials and whether they are elicited when target objects are not spatially segregated from nontargets. The present study was designed to address these questions.

Consider the pictures in Figure 1. Pictures A and $\mathrm{A}^{\prime}$ display a simple array of eight everyday objects that are identical, except for one critical difference: One object has been altered. If these pictures were flickered on the screen in quick succession, it would be fairly easy to detect the rotation of the cigarette lighter in the upper left corner. However, as was described above, such subtle changes become far more difficult to detect when a blank screen (i.e.,
A

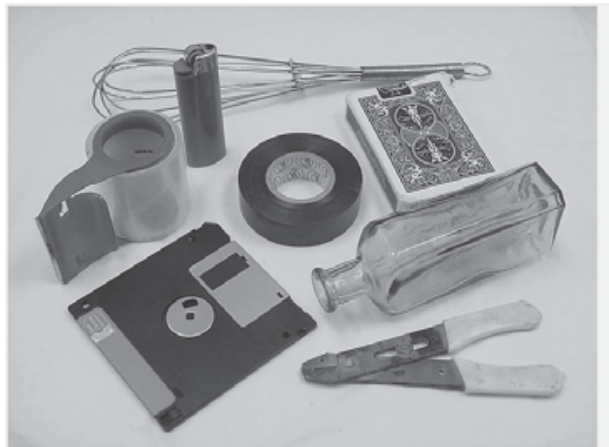

$A^{\prime}$

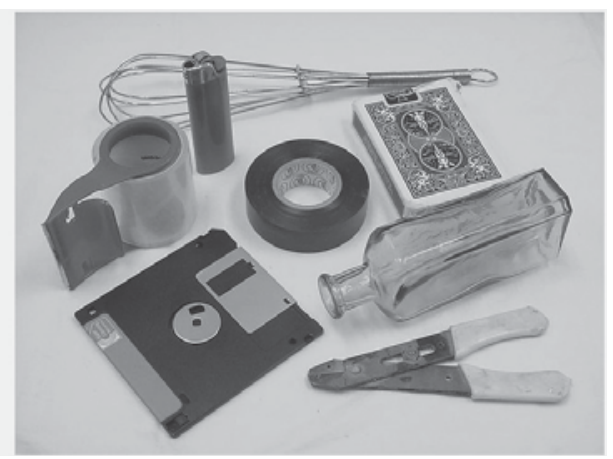

Figure 1. Stimuli from an example trial. Displays $A$ and $A^{\prime}$ would be shown in an alternating fashion, separated by a brief mask. The only difference between the two pictures is that one of the items has been rotated around its vertical axis. 
a global transient) is interleaved between the presentations of each picture, because it masks the motion cues that are commonly used to detect change. We hypothesized that the ability to detect such changes would be influenced by the type of display (smoking related vs. unrelated), the participants' experience with regard to the target objects (smokers vs. nonsmokers), and the participants' knowledge about the nature of the experiment (awareness vs. unawareness of smoking focus). Type of participant could be considered a proxy for a long-term, relatively contextindependent attentional bias. Smokers are likely to have an attentional bias for smoking-related objects, irrespective of where they are. Awareness of the nature of the experiment could be considered a proxy for short-term, contextdependent attentional bias. That is, nonsmokers are not likely to have a long-term attentional bias for smokingrelated objects but might temporarily develop one in the context of an experiment on smoking. The present design allowed us to examine the effects of each of these two types of biases on change detection, as well as to address whether these biases have additive effects.

We presented three types of randomly arranged scenes of eight objects to each participant. The first type of scene, smoking changed, contained one smoking item, which was rotated by $180^{\circ}$ between the two versions of the picture; all the other items remained unchanged. The smokingunchanged scenes also contained a smoking item, but here it was one of the other items that was rotated between the two versions of the picture. The smoking-absent scenes, finally, did not contain any smoking items, but one of the items was rotated between the two versions of the picture. We predicted that attentional biases would differentially affect the observers' ability to detect change. An attentional bias for smoking objects should lead to shorter detection latencies when the smoking object changed (because attention was likely to be focused on the changing item) and longer when it did not change (because attention was more likely to be focused on the nonchanging smoking item and, thus, less likely to be focused on the changing nonsmoking item; see also B. T. Jones et al., 2003). If only a long-term context-independent bias produces such an effect, it should be manifest only in smokers. However, if a short-term context-dependent attentional bias developed in nonsmokers who were aware of the purpose of the experiment, they should also exhibit the effect. In contrast, nonsmokers unaware of the experiment's focus should not respond differentially to smoking and nonsmoking items. Finally, if the long-term and short-term attentional biases have additive effects, the described pattern should be stronger in smokers aware of the experimental focus than in smokers not aware of this focus. However, if attentional bias is an all-or-none phenomenon, the two groups should perform equivalently. To manipulate awareness of experimental focus, we informed half of the smokers and half of the nonsmokers that they were participating in a smoking and attention study and the other half that they were participating in an attention study; that is, we did not inquire about their smoking status until after the flicker task had been completed.

\section{METHOD}

\section{Participants}

Sixty undergraduate students enrolled at Florida State University participated. Forty-five of the students were enrolled in introductory psychology courses and participated for course credit. The remaining 15 students participated for monetary compensation. For the aware condition, we advertised for smokers and nonsmokers, whereas in the unaware condition, we simply inquired about smoking status after the experiment had been completed. The participants were considered smokers if they smoked at least 10 cigarettes per day for a period of at least 12 months. All smoking-related questions were asked prior to the experiment in the aware condition and after the experiment in the unaware condition.

\section{Materials}

Thirty-two black-and-white picture pairs were constructed. In each picture, an array of eight objects was randomly arranged on a white background. The objects included smoking objects (e.g., matches, a cigarette, and a lighter) and nonsmoking objects (e.g., a screwdriver, a wrench, and a diskette). Given the specific perceptual features of the smoking-related objects, it was difficult to match them with nonsmoking counterparts that were clearly unrelated to smoking. Therefore, the objects were selected to be categorically related or unrelated to smoking activities. Although there was variety in the shapes, sizes, and textures of the objects, all of them were objects that would commonly be found in a kitchen (e.g., a spoon), in an office (e.g., a roll of tape), or on a workbench (e.g., pliers). Object groups were randomly selected for each scene. Although some of the objects were used in multiple scenes, none of the target objects was repeated. The pictures were converted to black and white in order to prevent color salience from capturing attention and aiding identification. All the (digital) pictures were scaled to $640 \times 480$ pixels. When presented at a $1,280 \times 1,024$ pixel screen resolution, the pictures were approximately $9 \times 7$ in.

Of the 32 picture pairs, 16 contained one smoking object with seven nonsmoking objects, and 16 contained eight nonsmoking objects (smoking absent). Eight of the smoking object pictures included the change of a smoking object (smoking changed), whereas the remaining eight included the change of a nonsmoking object (smoking unchanged). The critical change always included the rotation of one object along its vertical or horizontal axis.

\section{Design and Procedure}

Four participant groups saw a randomized presentation of the 32 experimental picture pairs. The four groups consisted of 15 smokers informed that they were participating in a smoking study, 15 smokers informed that they were participating in an attention study, 15 nonsmokers informed that they were participating in a smoking study, and 15 nonsmokers informed that they were participating in an attention study (1 participant's data were excluded from the analyses, due to group mislabeling). Thus, half of the participants were aware that the study was related to smoking, whereas the remaining half was not. Group was a between-participants variable, and object type was a within-participants and item variable.

The experiment was run on PCs with 19-in. flat-screen displays, using the E-Prime stimulus presentation software (Schneider, Eschman, \& Zuccolotto, 2002). During each trial, a fixation point appeared in the center of the screen for $500 \mathrm{msec}$, after which the pictures were presented in series: Picture A, blank screen, Picture A', blank screen, and so on. The pictures were presented for $80 \mathrm{msec}$, and blank screens were presented for $500 \mathrm{msec}$. The participants 
were instructed to identify the object that changed in the flickering pictures and, upon detecting the change, to press the space bar as quickly as possible. To ensure that the correct object was identified, the participants had to type the name or a brief description of the object that changed after each trial. Furthermore, the participants were instructed to keep their fingers on the space bar to ensure timely responses as reaction times were being measured. The picture presentation sequence was designed to end when the participant pressed the space bar or when the 35 -sec time limit was reached. The experiment took approximately $20 \mathrm{~min}$ to complete.

\section{RESULTS}

Number of scene alternations and error rates of experimental trials were submitted to a 2 (smoking status) $\times$ 2 (awareness of smoking relatedness) $\times 3$ (object type) ANOVA with repeated measurement on object type. The alternation analysis was performed on correct responses only. Responses greater than two standard deviations from the participant's mean in the respective condition were ommitted from the analysis (this removed less than $6 \%$ of the data). The mean detection alternations and standard deviations are displayed in Table 1.

In all the analyses reported below, the alpha level was set at $p=.05$. Most relevant to our predictions, there was a significant three-way interaction between smoking status, awareness, and object type $\left[F(2,95)=3.51, M S_{\mathrm{e}}=\right.$ 37 , using the Greenhouse-Geisser correction for the violation of sphericity assumption]. We conducted followup analyses to examine the effect of smoking status and awareness. There was no interaction between object type and awareness for smokers $[F(2,45)<1]$. However, for smokers, there was a main effect of object type $[F(2,45)=$ $\left.6.30, M S_{\mathrm{e}}=78\right]$. To investigate the main effect of object type for smokers, we performed a paired-samples $t$ test on object type, using the Bonferroni correction to control for the number of comparisons. Responses to smokingchanged items were significantly faster than those to smoking-unchanged items $\left[t(29)=-2.85, M S_{\mathrm{e}}=0.98\right]$. Also, responses to smoking-absent items were significantly faster than those to smoking-unchanged items $\left[t(29)=-2.87, M S_{\mathrm{e}}=0.69\right]$.

Unlike for smokers, there was an interaction of object type and awareness for nonsmokers $[F(2,54)=5.60$, $\left.M S_{\mathrm{e}}=46\right]$. To investigate this interaction further, we examined the effect of awareness. For aware nonsmokers,

Table 1

Mean Alternations (With Standard Deviations) of Correct Responses in the Change Detection Task

\begin{tabular}{|c|c|c|c|c|c|c|}
\hline & \multicolumn{2}{|c|}{$\begin{array}{c}\text { Smoking } \\
\text { Absent }\end{array}$} & \multicolumn{2}{|c|}{$\begin{array}{l}\text { Smoking } \\
\text { Changed }\end{array}$} & \multicolumn{2}{|c|}{$\begin{array}{c}\text { Smoking } \\
\text { Unchanged }\end{array}$} \\
\hline & $M$ & $S D$ & $M$ & $S D$ & $M$ & $S D$ \\
\hline \multicolumn{7}{|l|}{ Smokers } \\
\hline Informed & 11.3 & 2.2 & 10.8 & 3.4 & 12.9 & 4.4 \\
\hline Naive & 10.9 & 3.3 & 9.9 & 3.9 & 13.4 & 5.4 \\
\hline \multicolumn{7}{|l|}{ Nonsmokers } \\
\hline Informed & 11.6 & 2.4 & 10.1 & 2.0 & 12.4 & 3.5 \\
\hline Naive & 10.4 & 2.6 & 13.8 & 4.9 & 12.6 & 5.3 \\
\hline
\end{tabular}

there was a main effect of object type $[F(2,28)=4.21$, $\left.M S_{\mathrm{e}}=20\right]$. Responses to smoking-changed items were significantly faster than those to smoking-unchanged items $\left[t(14)=-2.69, M S_{\mathrm{e}}=0.84\right]$, and responses to smoking-changed items were significantly faster than those to smoking-absent items $\left[t(14)=2.20, M S_{\mathrm{e}}=0.67\right]$. For unaware nonsmokers, there was also a main effect of object type $\left[F(2,26)=3.51, M S_{\mathrm{e}}=42\right]$. Responses to smoking-absent items were significantly faster than those to smoking-changed items $\left[t(13)=-2.80, M S_{\mathrm{e}}=1.22\right]$, and responses to smoking-absent items were marginally significantly faster than those to smoking-unchanged items $\left[t(13)=-1.90, M S_{\mathrm{e}}=1.16, p<.08\right]$. Thus, the aware nonsmokers behaved like the smokers, whereas the unaware nonsmokers did not. ${ }^{1}$ To investigate the effect of awareness for each object type, we performed an independent samples $t$ test. Aware nonsmokers responded to smoking change items significantly more quickly than did unaware nonsmokers $[t(27)=2.67]$. Also, unaware smokers responded more quickly to the smoking-changed items than did unaware nonsmokers $[t(27)=-2.34]$, whereas there were no differences between the aware smokers and the nonsmokers for any of the object types $[t(28)<1]$.

For detection alternations, there was also a significant main effect of object type [smoking absent vs. smoking changed vs. smoking unchanged, $F(2,95)=6.28, M S_{\mathrm{e}}=$ 66 , using the Greenhouse-Geisser correction for the violation of sphericity assumption]. The smoking-changed items were responded to more quickly than were the smoking-unchanged items $[t(58)=-2.53]$, and smokingabsent items were also responded to more quickly than were the smoking-unchanged items $[t(58)=-3.51]$. Overall, the smoking-absent and smoking-changed items elicited the shortest detection rates (11.1), whereas the smoking-unchanged items elicited the longest (12.8). Given that the items were not matched along perceptual dimensions but, rather, were categorized according to their smoking relationship, it is difficult to interpret this overall pattern without referring to the overall influence of smoking status and awareness on object type.

Accuracy rates were also submitted to a $2 \times 2 \times 3$ ANOVA. Because many of the accuracy scores were perfect, the accuracy scores were normalized using the arcsine transformation before analysis. There was a significant main effect of object type [smoking absent vs. smoking changed vs. smoking unchanged, $F(2,98)=6.25, M S_{\mathrm{e}}=0.39$, using the Greenhouse-Geisser correction for the violation of sphericity assumption]. To investigate this main effect further, we performed a paired-samples $t$ test on the accuracy scores. Smoking-changed items were detected more accurately than were smoking-absent items $[t(58)=-2.72]$, and smoking-changed items were also detected more accurately than were smoking-unchanged items $[t(58)=2.92]$. Overall, the smoking-changed items were detected most accurately (1.45), the smoking-unchanged items were detected least accurately (1.30), and the smoking-absent items were in between (1.33). Thus, the smoking-unchanged items elicited the longest detection times and the poorest 
accuracy. It seems that overall, the presence of an unchanging smoking object grabbed the individuals' attention and caused them to evaluate the scenes longer but did not necessarily lead to a more successful completion of the task. This general pattern holds for all but the unaware nonsmokers. All the remaining interactions were nonsignificant [object type $\times$ awareness, $F(2,98)=2.27, p<.16$; object type $\times$ smoking, $F<1$; and object type $\times$ smoking $\times$ awareness, $F(2,98)=2.20, p<.12]$.

\section{DISCUSSION}

The results suggest that both long-term and short-term attentional bias affected detection latencies. Long-term attentional biases were manifest in the performance of the two groups of smokers, whose change detection latencies were shortest when a smoking-related object changed and longest when a smoking-related object was present but did not undergo change. Short-term attentional bias was manifest in the finding that the aware nonsmokers - that is, those with a short-term bias - performed like the smokers, whereas the unaware nonsmokers showed no bias toward smoking-related items. The fact that the two groups of smokers were statistically indistinguishable suggests that attentional bias is an all-or-none phenomenon, at least in the context of a change detection paradigm. If there had been an additive or subadditive effect, the aware smokers' bias should have been more pronounced than that of the unaware smokers. In addition, these data suggest that attention was not simply attracted by an intrinsic difference in saliency between the smoking and the neutral objects but, rather, was modulated differentially as a function of the participants' long-term and short-term attentional biases.

The findings suggest that the two types of attentional bias influence change detection to a similar degree, given that the performances of the unaware smokers and the aware nonsmokers were statistically indistinguishable. More empirical evidence is needed before firm conclusions can be drawn about the relative effects of contextindependent and context-dependent attentional bias. However, our results clearly demonstrate that both affect the ability to detect change in the environment. Thus, it appears that the flicker paradigm is a suitable tool for investigating a variety of attentional biases, in that it is sensitive enough to detect the influence of biases within an individual trial (B. C. Jones et al., 2002; B. T. Jones et al., 2003) or across multiple trials, as well as sensitive enough to detect the influence of biases when objects are not categorically segregated in a scene. To determine how suitable the flicker paradigm is as a diagnostic tool, it will be important to determine how levels of use, addiction, and deprivation influence the ability to detect change, as well as how generalizable such findings are for various types of contexts and scene depictions.

\section{REFERENCES}

Ehrman, R. N., Robbins, S. J., Bromwell, M. A., Lankford, M. E., Monterosso, J. R., \& O'Brien, C. P. (2002). Comparing attentional bias to smoking cues in current smokers, former smokers, and nonsmokers using a dot-probe task. Drug \& Alcohol Dependence, 67, 185-191.

Jones, B. C., Jones, B. T., Blundell, L., \& Bruce, G. (2002). Social users of alcohol and cannabis who detect substance-related changes in a change blindness paradigm report higher levels of use than those detecting substance-neutral changes. Psychopharmacology, 165, 93-96.

Jones, B. T., Jones, B. C., Smith, H., \& Copley, N. (2003). A flicker paradigm for inducing change blindness reveals alcohol and cannabis information processing biases in social users. Addiction, $\mathbf{9 8}$, 235-244.

Levin, D. T., \& Simons, D. J. (1997). Failure to detect changes to attended objects in motion pictures. Psychonomic Bulletin \& Review, 4, 501-506.

MadDEN, C. J., \& ZwaAn, R. A. (2001). The impact of smoking urges on working memory performance. Experimental \& Clinical Psychopharmacology, 9, 418-424.

MCConkie, G. W., \& CurRie, C. B. (1996). Visual stability across saccades while viewing complex pictures. Journal of Experimental Psychology: Human Perception \& Performance, 22, 563-581.

Mogg, K., Bradley, B. P., Field, M., \& De Houwer, J. (2003). Eye movements to smoking-related pictures in smokers: Relationship between attentional biases and implicit and explicit measures of stimulus valence. Addiction, 98, 825-836.

O’Regan, J. K. (1992). Solving the "real" mysteries of visual perception: The world as an outside memory. Canadian Journal of Psychology, 46, 461-488.

O'Regan, J. K., \& NoË, A. (2001). A sensorimotor account of vision and visual consciousness. Behavioral \& Brain Sciences, 24, 939-1031.

Rensink, R. A. (2000). The dynamic representation of scenes. Visual Cognition, 7, 17-42.

Rensink, R. A. (2002). Change detection. Annual Review of Psychology, 532, 245-277.

Rensink, R. A., O'Regan, J. K., \& Clark, J. J. (1997). To see or not to see: The need for attention to perceive changes in scenes. Psychological Science, 8, 368-373.

RYAN, F. (2002). Detected, selected, and sometimes neglected: Cognitive processing of cues in addiction. Experimental \& Clinical Psychopharmacology, 10, 67-76.

SayetTe, M. A., \& HufFord, M. R. (1994). Effects of cue exposure and deprivation on cognitive resources in smokers. Journal of Abnormal Psychology, 103, 812-818.

Schneider, W., Eschman, A., \& Zuccolotto, A. (2002). E-Prime reference guide. Pittsburgh: Psychology Software Tools.

Scholl, B. J. (2000). Attenuated change blindness for exogenously attended items in a flicker paradigm. Visual Cognition, 7, 377-396.

Simons, D. J., \& Levin, D. T. (1997). Change blindness. Trends in Cognitive Sciences, 1, 261-267.

Tiffany, S. T. (1990). A cognitive model of drug urges and drug-use behavior: Role of automatic and nonautomatic processes. Psychological Review, 97, 147-168.

ZwaAn, R. A., \& TruitT, T. P. (1998). Smoking urges affect language processing. Experimental \& Clinical Psychopharmacology, 6, 325-330.

ZwaAn, R. A., \& Truitt, T. P. (2000). Inhibition of smoking-related information in smokers and nonsmokers. Experimental \& Clinical Psychopharmacology, 8, 192-197.

\section{NOTE}

1. The signature of the bias for smoking-related objects is relatively short detection times for scenes in which the smoking object changes and relatively long detection times for scenes in which a smoking object is present but a different object changes. To assess how this bias emerged 
over the course of the experiment, we segregated each participant's data into two blocks of trials (from the first and second halves of the task). If the naive participants developed sensitivity to the stimulus contingencies over the course of the experiment, they should be more likely to display this bias in the second half of the experiment than in the first half. For naive nonsmokers, the reverse trend appeared. In the first block, their responses were 0.7 alternations faster on smoking-changed trials than on smoking-unchanged trials. In the second block, however, their responses were 2.7 alternations slower on smoking-changed trials than on smoking-unchanged trials. Naive smokers also showed no sign of having learned to attend to the smoking-related item in the dis- play. Their responses in the first block were 3.6 alternations faster on smoking-changed trials than on smoking-unchanged trials, and in the second block this advantage for smoking-changed trials fell to only 1.0 alternation. For comparison, informed participants in the second block of trials responded more quickly for smoking-changed trials than for smoking-unchanged trials (2.6 alternations for informed smokers and 1.3 for informed nonsmokers).

(Manuscript received May 14, 2004;

revision accepted for publication April 29, 2005.) 